Document downloaded from:

http://hdl.handle.net/10251/102285

This paper must be cited as:

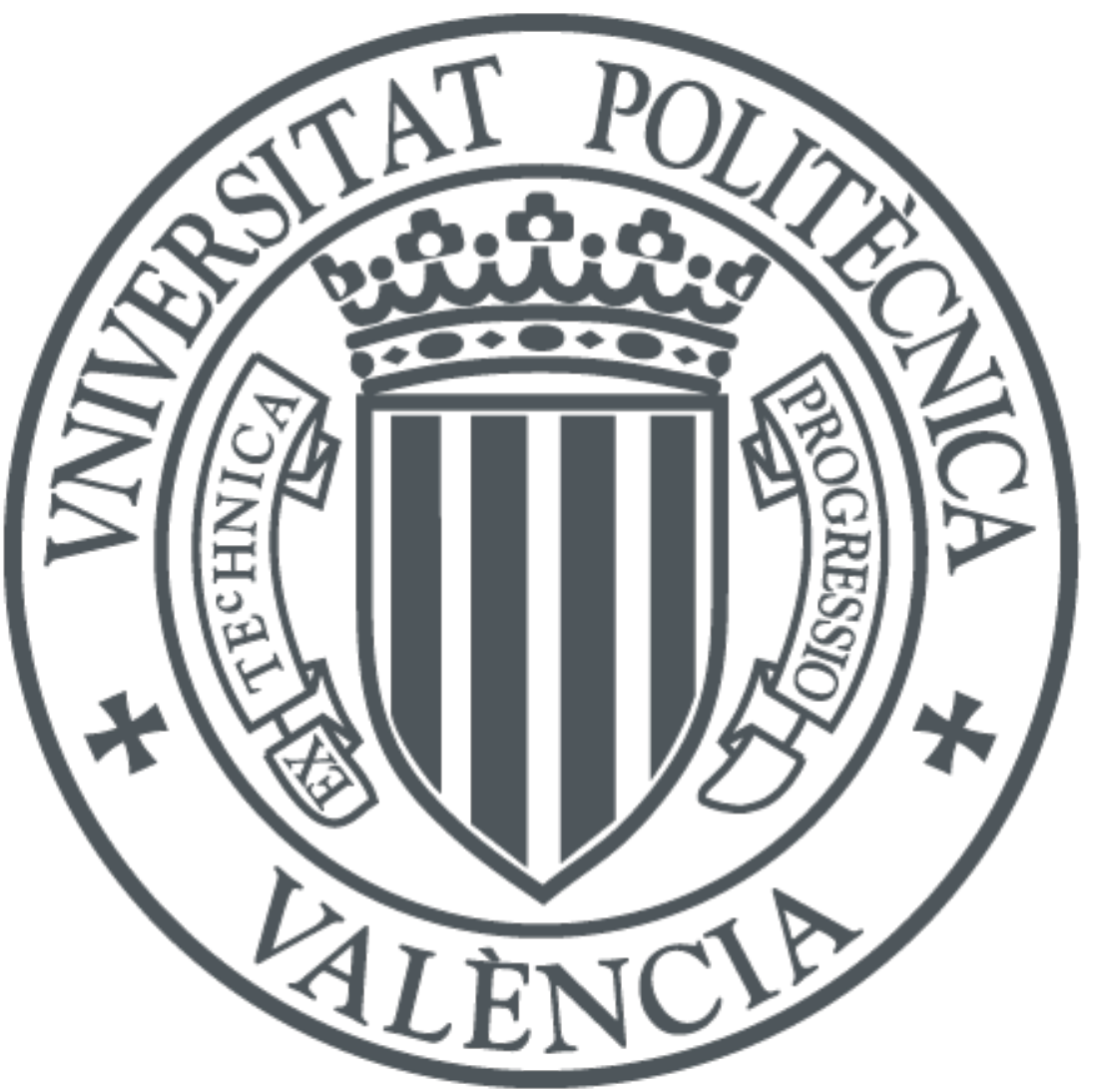

The final publication is available at

http://doi.org/10.1109/LPT.2017.2668464

Copyright Institute of Electrical and Electronics Engineers

Additional Information 


\title{
OOFDM signal transmission using a single optical broadband source
}

\author{
F. I. Chicharro, B. Ortega, Member, IEEE, M. de Diego, Member, IEEE, and J. Mora
}

\begin{abstract}
In this paper, optical OFDM signal transmission is demonstrated by using a broadband optical source over a $10 \mathrm{~km}$ fiber link. OFDM signal transmission usually employs lasers to generate the optical carrier since the chromatic fiber dispersion prevents the use of broadband optical sources. However, an interferometric system is introduced to allow signal transmission at a given RF band when a broadband optical source is employed. Optical sources with different optical bandwidths have been employed to explore the limitations of the system. OFDM transmission at $2 \mathrm{~Gb} / \mathrm{s}$ using both QPSK and 16QAM modulation is demonstrated over $10 \mathrm{~km}$.
\end{abstract}

Index Terms - Optical fibers, Optical networks, OFDM, broadband sources.

\section{INTRODUCTION}

$\mathrm{D}$ uring the past several decades, the exponential growth of traffic on long-haul, metropolitan and access networks has led to an increased need for bandwidth. Orthogonal Frequency Division Multiplexing (OFDM) modulation is a suitable technology for low-cost, high-capacity networks. Optical OFDM (OOFDM) transmission has attracted considerable research interest, as OFDM is already successfully used in several wireless and wireline standards [1, 2]. Its high spectral efficiency and its advantages in overcoming transmission impairments such as chromatic and polarization mode dispersion [1] are the main reasons for its wide deployment.

Depending on the optical receiver structure, OOFDM can use either direct or coherent detection. The former is the simplest approach for optical OFDM systems and is especially suitable for metropolitan and access networks [3], whereas the latter offers better sensitivity and spectral efficiency but higher complexity and costs [4]. The generation of intensitymodulated and directly detected (IM/DD) OFDM signals employs either double-sideband (DSB) modulation, which occupies a larger bandwidth in the optical spectrum and

Manuscript received -, -; revised -, -; accepted -, -. Date of publication -, -; date of current version -, -. This work was supported by the National project MINECO TEC2014-60378-C2-1-R MEMES and the Regional GVA funded Prometeo 2013/012 project on Next Generation Microwave Photonic technologies.

F.I. Chicharro, B. Ortega, M. de Diego and J.Mora are with the Instituto de Telecomunicaciones y Aplicaciones Multimedia (ITEAM) at Universitat Politècnica de València. Valencia 46022, Spain. (e-mails: frachilo@upvnet.upv.es, bortega@dcom.upv.es, mdediego@dcom.upv.es, jmalmer@iteam.upv.es). suffers from chromatic dispersion induced power fading, or single-sideband (SSB), which is preferentially employed due to its lack of power fading and more efficient usage of the spectrum at the expense of the SSB modulation complexity [5].

Wavelength-division-multiplexed (WDM) OFDM systems based on IM-DD require the use of optical multicarrier sources on the transmitter side, such as a semiconductor mode-locked laser, a gain-switched externally injected distributed feedback laser or an electro-optic modulator based comb source [6]. Several papers in the literature report on the laser noise requirements of distributed feedback (DFB) lasers [7, 8] and multicarrier sources [9] for guaranteeing good performance of directly detected OOFDM systems.

However, no papers have reported on the usage of optical broadband sources for transmitting OFDM signals over optical fibers, despite their cost-effectiveness and robustness to the optical crosstalk and temperature drift of other WDM components. A subcarrier multiplexing tolerant dispersion transceiver based on optical broadband sources was presented in [10] and offers the prospect of radio over fiber (RoF) signal transmission by using spectrum-sliced incoherent light sources such as amplified spontaneous emission (ASE) or lightemitting diodes (LEDs) in WDM networks.

In this paper, we propose, for the first time to our knowledge, a low-cost OOFDM signal transmitter based on an optical broadband source for moderate bitrate signal transmission in WDM access networks that is also compatible with multi-band next-generation OFDM-PON schemes [11]. Such a system would also serve for LANs based on MMF links [12].

The paper is structured as follows: section II describes the proposed system, section III presents the experimental setup and section IV reports the experimental transmission results. Finally, section $\mathrm{V}$ summarizes the main conclusions of the paper.

\section{SYSTEM DESCRIPTION}

Consider the optical signal emitted by a broadband optical source with power spectral distribution $S(\omega)$, which is amplitude-modulated by a DSB modulation in an electrooptic modulator and transmitted over an optical fiber link of length $L$ and dispersion parameter $\beta_{2}$. The electrical transfer function $H^{R F}(\Omega)$ can be calculated by [10]

$$
H^{R F}(\Omega) \propto C S E(\Omega) \cdot H_{O}^{R F}(\Omega)
$$




$$
\operatorname{CSE}(\Omega)=\cos \left(\frac{1}{2} \beta_{2} L \Omega^{2}\right)
$$

is the carrier suppression effect, which leads to power fading, caused by the phase difference of the sidebands due to fiber propagation [5]. The term $H_{0}^{R F}(\Omega)$ limits the range of the operating frequencies due to the low-pass filtering response obtained when a typical Gaussian optical spectrum of a certain bandwidth $\delta \omega_{3 d B}$ is considered. This term can be calculated as follows:

$$
H_{o}^{R F}(\Omega)=\frac{\int S(\omega) \cdot \exp \left\{-j \beta_{2} L\left(\omega-\omega_{0}\right) \Omega\right\} \cdot d \omega}{\int S(\omega) \cdot d \omega}
$$

This function is significantly modified when a MachZehnder interferometer (MZI) under a coherent regime is introduced in the transmission link, resulting, in this case, in the following transfer function:

$$
\begin{aligned}
& H^{R F}(\Omega) \propto\left\{\cos \left(\Omega \frac{\Delta \tau}{2}\right) \cdot C S E \cdot H_{o}^{R F}(\Omega)+\right. \\
& \left.+\frac{1}{2} \cdot C S E_{\mathrm{mod}}^{-} \cdot H_{o}^{R F}\left(\Omega-\Omega_{o}\right)+\frac{1}{2} \cdot C S E_{\mathrm{mod}}^{+} \cdot H_{o}^{R F}\left(\Omega+\Omega_{o}\right)\right\}
\end{aligned}
$$

where $\Delta \tau=\tau_{1}-\tau_{2}$ is the differential delay between the interferometer fiber arms, and the term $\operatorname{CSE}_{\text {mod }}^{\mp}$ is a frequency-shifted version of the CSE term whose impact at $\Omega_{0}$ is, therefore, negligible [10]:

$$
C S E_{\bmod }^{\mp}=\cos \left(\frac{1}{2} \beta_{2} L \Omega\left(\Omega \mp \Omega_{o}\right)\right)
$$

In this case, a new term $H_{0}^{R F}\left(\Omega \mp \Omega_{0}\right)$ appears, and a new RF transmission window is defined. It features the same structure as the baseband but is centered at $\Omega_{0}$ :

$$
\Omega_{o}=\frac{\Delta \tau}{\beta_{2} L}
$$

Note that the carrier suppression effect has been avoided in a DSB conventional modulation scheme, which also simplifies the modulation stage.

\section{EXPERIMENTAL SETUP}

Fig. 1 presents a schematic of the experimental setup. The optical broadband source is an NP Photonics C\&L Band ASE Source emitting $19.5 \mathrm{dBm}$ optical power with a $0.01 \mathrm{~dB} /$ hour stability in the $1525-1610 \mathrm{~nm}$ spectral range, whose spectral bandwidth is varied by a Peleton Optical Channels Controller $(0.8-38.4 \mathrm{~nm})$. The output signal is modulated by a QPSK OFDM signal using an AVANEX electrooptic modulator. The OFDM generation block diagram is shown in inset (c) of Fig. 1. An IFFT size of 2048 with 172 data-bearing subcarriers satisfying Hermitian symmetry is used. After adding a $6.25 \%$ cyclic prefix, the data are loaded in the DAC of the arbitrary waveform generator (AWG7122C, Tektronix), sampling at 24 GS/s. A block-type pilot pattern is applied, where 90 symbols are data and 10 are pilots. The electrical signal of $1 \mathrm{GHz}$ bandwidth is centered at $5 \mathrm{GHz}$, resulting in a $2 \mathrm{~Gb} / \mathrm{s}$ raw data rate stream.

The optical modulated signal is transmitted through a $10 \mathrm{~km}$ SSMF link $\left(\beta_{2}=-20 \mathrm{ps}^{2} / \mathrm{km}\right)$, as depicted in Fig. 1 . The system

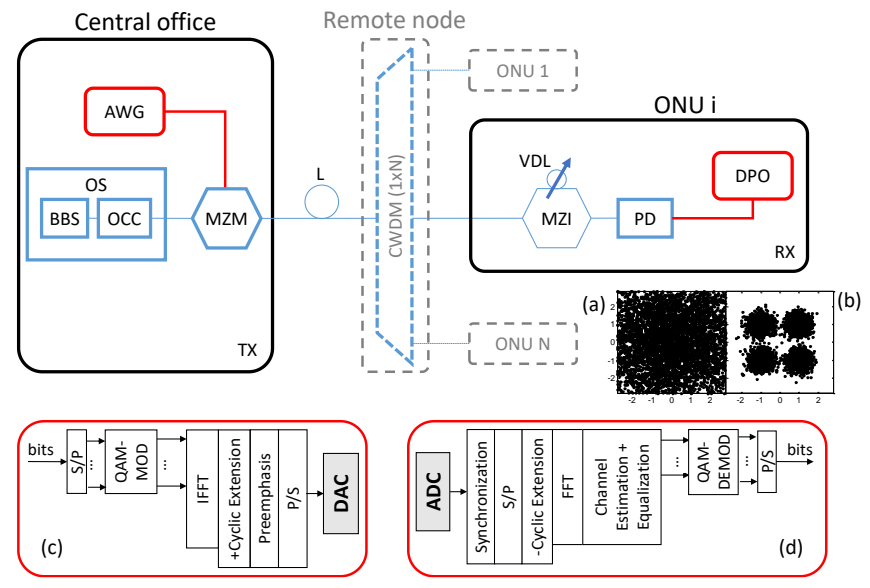

Fig. 1. OOFDM signal transmission experimental setup using a broadband optical source. BBS: Broadband Source, OCC: Optical Channel Controller, OS: Optical Source, MZM, Mach-Zehnder Modulator, AWG: Arbitrary Waveform Generator, CWDM: Coarse Wavelength Division Multiplexer, MZI: Mach-Zehnder Interferometer, VDL: Variable Delay Line, PD: Photodiode, ONU: Optical Network Unit, DPO: Digital Phosphor Oscilloscope. Insets: QPSK-OOFDM signal constellations after $10 \mathrm{~km}$ fiber signal transmission using a $3.9 \mathrm{~nm}$ bandwidth broadband optical source (a) without MZI module and (b) using the MZI module; block diagram of OFDM generation (c) and detection (d).

is compatible with a cost-effective WDM network based on a single source, where different broadband channels are generated at the CWDM by the spectrum slicing of the BBS output (an OCC is not required in this case). The channels are demultiplexed at a remote node before detection.

Prior to photodetection, and according to the previous section, the signal is transmitted through an MZI, which is composed of two 50:50 optical couplers and a variable delay line (VDL) in one of the arms. The VDL insertion loss is approximately $1 \mathrm{~dB}$, and its maximum delay range is $330 \mathrm{ps}$. Note that the signal constellation in inset (a) of Fig. 1 shows that QPSK OFDM signal transmission is not feasible using a $3.9 \mathrm{~nm}$ bandwidth optical source over a $10 \mathrm{~km}$ fiber link, according to the low-pass filtering effect described in (3). Indeed, the inset (b) of Fig. 1 shows the signal transmission when the interferometric structure is properly configured, as detailed in (4)-(6).

After photodetection, the received electrical signal is sampled at $50 \mathrm{GS} / \mathrm{s}$ by a real-time digital oscilloscope (DPO72004C, Tektronix), and the captured samples are synchronized and parallelized. The cyclic prefix is removed, and the FFT is applied. The block-type pilots are used for channel estimation and signal equalization. The data are demodulated, and the transmitted bit stream is recovered after the serialization stage. Inset (d) of Fig. 1 depicts the detection block diagram of the OFDM signal.

Fig. 2(a) shows the electrical amplitude response obtained when a laser and a $3.9 \mathrm{~nm}$ broadband source are employed as optical sources (black solid and blue dotted, respectively). The low-pass filtering response in the latter case limits the range of operating frequencies. However, as explained in the previous section, provided that an interferometric module is inserted with a delay of $\Delta \tau=6.28$ ps prior to the signal detection, a passband is encountered at $5 \mathrm{GHz}$ (green dashed trace), 
according to (6). The bandwidth is $1.13 \mathrm{GHz}$, and narrower bandwidths are obtained when optical sources with larger spectral bandwidths are employed. More concretely, Figs. 2(b) and 2(c) show the obtained transfer functions when optical sources with 7.09 and $10.28 \mathrm{~nm}$ optical bandwidths are employed, respectively, with and without the MZI module. The corresponding measured electrical bandwidths of the proposed transmission system are 0.75 and $0.67 \mathrm{GHz}$, respectively. In Fig. 2, all the graphs include the electrical spectrum of the OFDM modulating signal to clearly illustrate the radio frequency bandpass filtering that allows the OOFDM signal transmission when a broadband optical carrier is

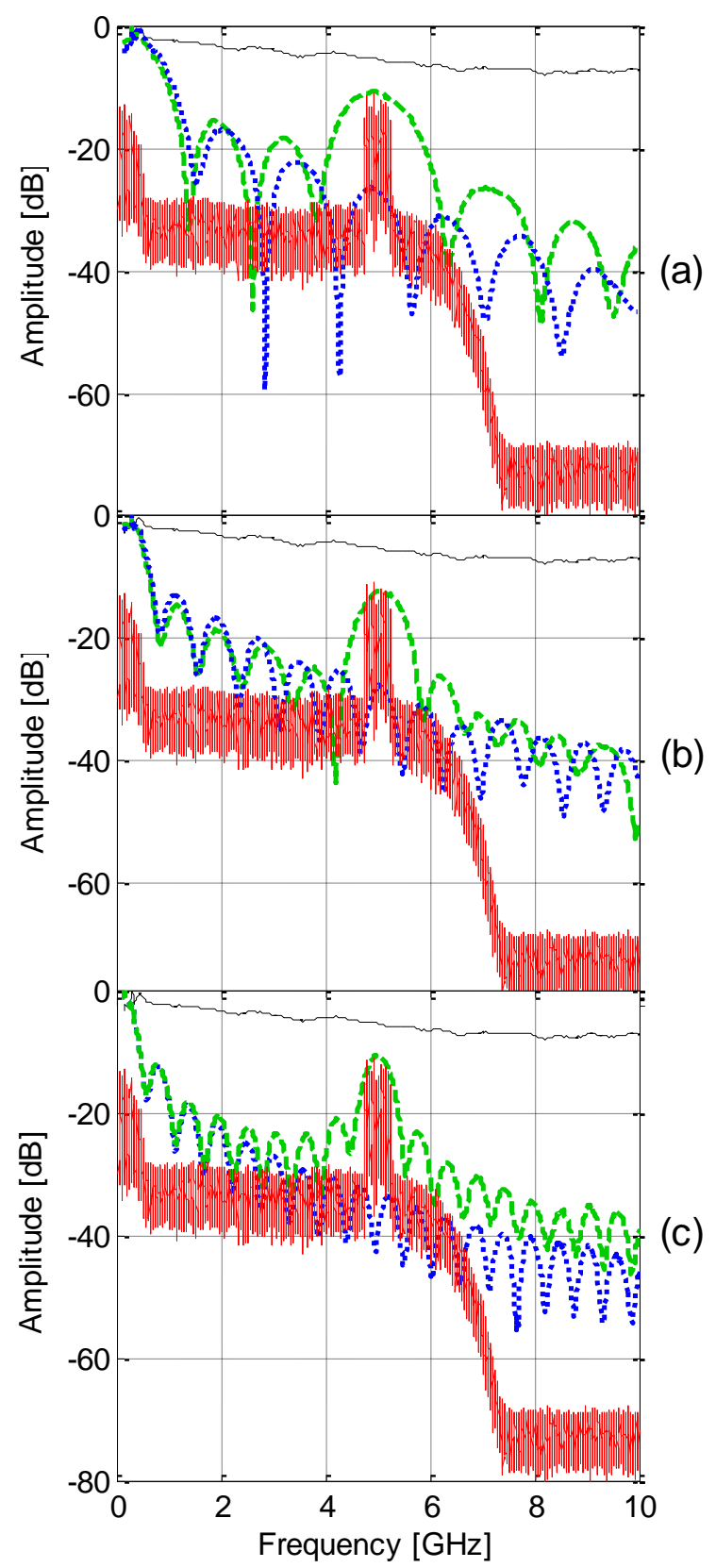

Fig. 2. Amplitude signal response after a $10 \mathrm{~km}$ fiber link using a laser (black line), a BBS (blue dotted line), a BBS with the system proposed (green dashed line) and the electrical modulating $1 \mathrm{GHz}$ bandwidth OFDM signal (red line). Measurements were done using OSs of different bandwidths: (a) $3.9 \mathrm{~nm}$, (b) $7.09 \mathrm{~nm}$ and (c) $10.28 \mathrm{~nm}$. employed with the proposed MZI module.

\section{TRANSMISSION RESULTS}

The link performance was tested with optical QPSK OFDM signals of different bandwidths by using optical sources with a $3 \mathrm{~dB}$ bandwidth of 3.9, 7.09 and $10.28 \mathrm{~nm}$. Fig. 3 shows the EVM value, measured as the power ratio between the symbol deviations and the transmitted symbols and obtained at a received power of $-6 \mathrm{dBm}$. As references, B2B values have been included for the three cases. The bandwidth limitation after the fiber transmission link is observed to be more severe when the $10.28 \mathrm{~nm}$ is employed since the RF bandwidth is measured as $0.67 \mathrm{GHz}$ (Fig. 2(b)), and therefore the broader

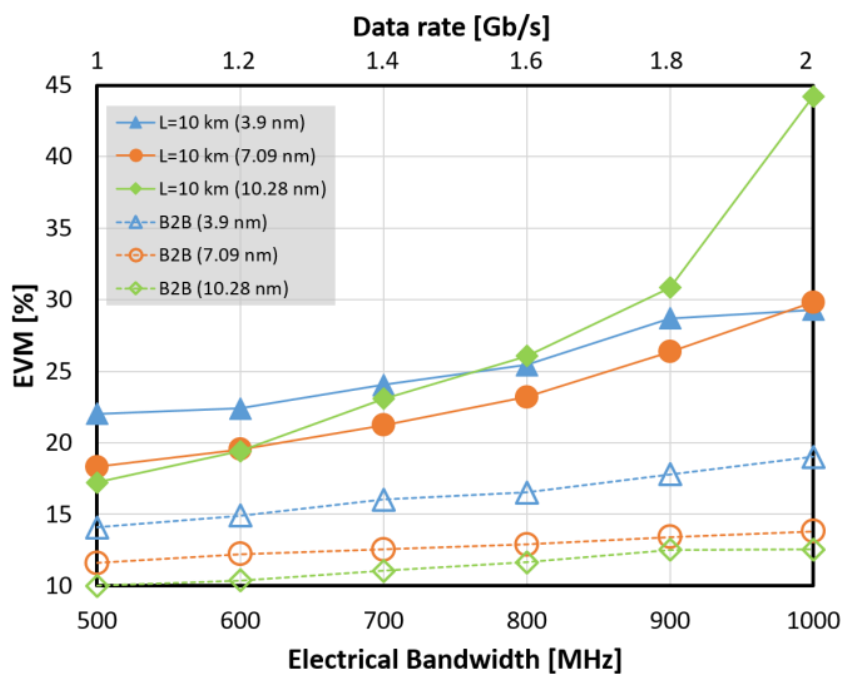

Fig. 3. Measured EVM for QPSK OFDM signals with different electrical bandwidths transmitted using OSs with different optical bandwidths: B2B (empty markers) and $10 \mathrm{~km}$ fiber link (solid markers).

band signal transmission is prevented. Although the degradation of the signal is larger when broader electrical bandwidth signals are transmitted, both the 3.9 and $7.09 \mathrm{~nm}$ optical sources allow the transmission of $1 \mathrm{GHz}$ bandwidth signals with EVM levels below 30\%.

Fig. 4 shows the measured EVM and BER values after 10 $\mathrm{km}$ fiber transmission when an optical QPSK OFDM $1 \mathrm{GHz}$

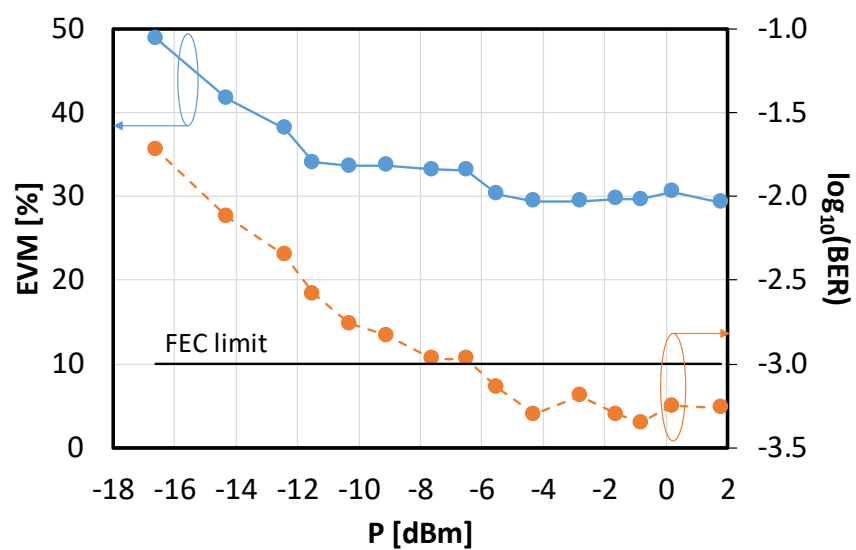

Fig. 4. Measured EVM (solid line) and BER (dashed line) values of OOFDM QPSK $1 \mathrm{GHz}$ signal transmitted over 10-km fiber link. 
signal is transmitted over a $5 \mathrm{GHz}$ RF signal by using a 3.9$\mathrm{nm}$ bandwidth optical source. As expected, the reduction of the optical detected power leads to an increase in the signal penalty. In our experimental setup, a received minimum optical power of $-6.3 \mathrm{dBm}$ was required to obtain BER values below the FEC limit of $10^{-3}$ while keeping the EVM lower than $32 \%$.

Finally, a $2 \mathrm{~Gb} / \mathrm{s}$ OOFDM 16 QAM signal was transmitted over a $500-\mathrm{MHz}$ bandwidth using our experimental setup employing a laser as a reference and a 3.9-nm bandwidth optical signal without the proposed module and with it. Fig. 5 shows the measured EVM and BER values after the fiber link and the MZI module, and the insets show the corresponding signal constellations. The signal can be successfully transmitted with an EVM of $17.67 \%$ and a BER below the FEC limit of $3.8 \cdot 10^{-3}$ [13], provided that $-7 \mathrm{dBm}$ of optical power is received at the end of the $10 \mathrm{~km}$ fiber link.

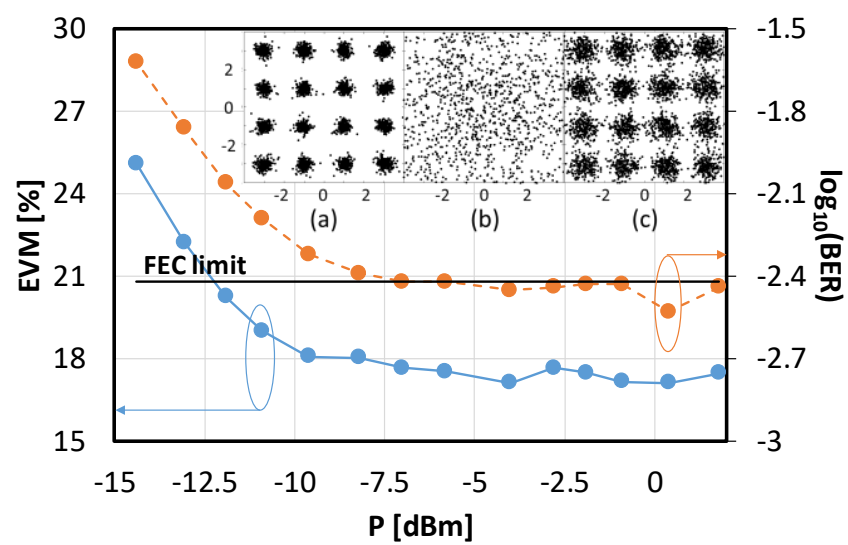

Fig 5. Measured EVM (solid line) and BER (dashed line) values of OOFDM 16-QAM $0.5 \mathrm{GHz}$ signal transmitted over $10 \mathrm{~km}$ fiber link. Insets: received constellations using a laser (a) and a $3.9 \mathrm{~nm}$ bandwidth optical source without (b) and with (c) the MZI module.

\section{CONCLUSIONS}

In conclusion, we have demonstrated optical OFDM transmission using an optical broadband source over a $10 \mathrm{~km}$ fiber link. A module based on an interferometric system is inserted prior to the detector to overcome the dispersion limitations of the system. Therefore, $2 \mathrm{~Gb} / \mathrm{s}$ DSB OOFDM transmission is feasible using a 3 to $7 \mathrm{~nm}$ bandwidth ASE optical source free of power fading. The proposal is easily adaptable to WDM access networks and compatible with multi-band next-generation OFDM-PONs. We are currently addressing the transmission of higher OOFDM bitrates with lower received optical powers to maximize performance and reduce the cost of next-generation optical access networks.

\section{REFERENCES}

[1] A. J. Lowery, J. Armstrong, "Orthogonal-frequency-division multiplexing for dispersion compensation of long-haul optical systems," Opt. Express, vol. 14, pp. 2079-2084, Mar 2006.

[2] N. Cvijetic, "OFDM for next generation optical access networks," $J$. Lightw. Technol., vol. 30, no. 4, pp. 384-398, Feb 2012.

[3] J. L. Wei, X. Q. Jin and J. M. Tang, "The Influence of Directly Modulated DFB Lasers on the Transmission Performance of CarrierSuppressed Single-Sideband Optical OFDM Signals Over IMDD SMF Systems," J. Lightw. Technol., vol. 27, no. 13, pp. 2412-2419, Jul 2009.

[4] W. Shieh, C. Athaudage, "Coherent optical orthogonal frequency division multiplexing," IEEE Elect. Lett., vol. 42, pp. 587-589, Feb 2006.

[5] B. Lin, J. Li, H. Yang, Y. Wan, Y. He, Z. Chen, "Comparison of DSB and SSB Transmission for OFDM-PON," J. Opt. Commun. Netw., vol. 4, no. 11, pp. B94-B100, Nov 2012.

[6] V. Vujicic, P. M. Anandarajah, C. Browning, L. P. Barry, "WDMOFDM-PON based on compatible SSB technique using a mode locked comb source," IEEE Photon. Technol. Lett., vol. 25, no. 21, pp. 20582061, Oct 2013.

[7] Z. Zan, M. Premaratne, A. J. Lowery, "Laser RIN and linewidth requirements for direct detection optical OFDM," in Proc. CLEO/QELS, San Jose, CA, USA, May 2008, pp. 1-2.

[8] W. R. Peng, "Analysis of laser phase noise effect in direct-detection optical OFDM transmission," J. Lightw. Technol., vol. 28, no. 17, pp. 2526-2535, Aug 2010.

[9] V. Vujicic, P. M. Anandarajah, R. Zhou, C. Browning, L. P. Barry, "Performance investigation of IM/DD compatible SSB-OFDM systems based on optical multicarrier sources," IEEE Photon. Journal, vol. 6, no. 5, pp. 1-11, Oct 2014.

[10] F. Grassi, J. Mora, B. Ortega, J. Capmany, "Subcarriers multiplexing tolerant dispersion transmission system employing optical broadband sources," Opt. Express, vol. 17, no. 6, pp. 4740-4751, Mar 2009.

[11] T. M. F. Alves, A. V. T. Cartaxo, "Power budget of ultra-dense virtualcarrier assisted DD MB-OFDM next-generation PON," IEEE Phot. Technol. Lett., vol. 28, no. 13, pp. 1406-1409, Mar 2016.

[12] F. Grassi, J. Mora, B. Ortega, J. Capmany, "GHz-multichannel SCMWDM transmission over multimode fiber links employing an optical broadband source", International Topical Meeting on Microwave Photonics. MWP '09, pp. 1 - 4 (2009).

[13] ITU-T Recommendation G.975.1, Appendix I.9 (2004). 